\title{
Sequential Lottery Contests with Multiple Participants
}

Max Planck Institute for Tax Law and Public Finance

Working Paper 2017 - 02

April 2017

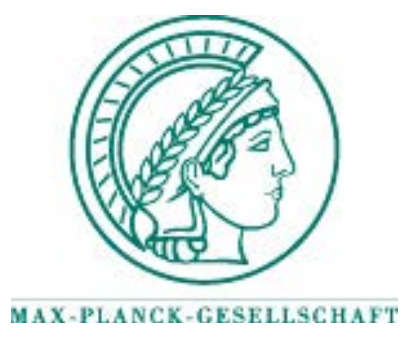

Max Planck Institute for

Tax Law and Public Finance

Department of Business and Tax Law

Department of Public Economics

http:/ / www.tax.mpg.de 
Working papers of the Max Planck Institute for Tax Law and Public Finance Research Paper Series serve to disseminate the research results of work in progress prior to publication to encourage the exchange of ideas and academic debate. Inclusion of a paper in the Research Paper Series does not constitute publication and should not limit publication in any other venue. The preprints published by the Max Planck Institute for Tax Law and Public Finance represent the views of the respective author(s) and not of the Institute as a whole. Copyright remains with the author(s).

Max Planck Institute for Tax Law and Public Finance

Marstallplatz 1

D-80539 Munich

Tel: $\quad+498924246-0$

Fax: $\quad+498924246-501$

E-mail: ssrn@tax.mpg.de

http://www.tax.mpg.de 


\title{
Sequential Lottery Contests with Multiple Participants
}

\author{
Nava Kahana ${ }^{a, b}$ Doron Klunover ${ }^{*}$
}

April 2017

\begin{abstract}
The literature on aggregative games, which has been applied in the study of contests, has focused on simultaneous games. We apply aggregative games techniques in a novel fashion in the analysis of sequential lottery contests with $n$ players. It is shown that: (1) there exists a unique subgame perfect equilibrium in pure strategies, and (2) unlike in the case of a small contest, aggregate expenditure in a large contest is lower than in the corresponding simultaneous contest.
\end{abstract}

Keywords: Aggregative games; Sequential Contests; Subgame Perfect Equilibrium.

JEL classification numbers: C72, D43, L13.

\footnotetext{
${ }^{a}$ Department of Economics, Bar-Ilan University, 52900 Ramat-Gan, Israel

b IZA, Bonn

* Corresponding author: Doron.Kluhovrd@biu.ac.il
} 


\section{Introduction}

In an imperfectly discriminative contest, there is a probabilistic relation between players' investments and prize allocation. ${ }^{1}$ A lottery contest is a special yet commonly used imperfectly discriminative contest with a functional form for winning in which the expenditures of the players are equivalent to tickets bought at a unit cost and the winner is drawn from the set of tickets sold (Wärneryd 2003; Konrad 2009). The lottery functional form has been axiomatized by Skaperdas (1996). Applications of the lottery contest include rent seeking (Tullock 1980; Hillman 2013), R\&D contests (Fullerton and McAfee 1999) and conflicts between groups (see, for example, Esteban and Ray 2011).

The literature on imperfectly discriminative sequential contests has focused mainly on the two-player case (see Dixit 1987; Baik and Shogren 1992; Leininger 1993; Linster 1993; Leininger and Yang 1994; Morgan 2003; Yildirim 2005; Morgan and Vardy 2007; Serena 2017). For this case, it has been shown that the Stackelberg and Nash solutions are identical when players are symmetric (Dixit 1987).

In the context of imperfectly discriminative sequential contests with more than two players, Dixit (1987) has discussed a contest with one leader and many parallel responders. Glazer and Hassin (2000) provided an analytical Stackelberg solution for a sequential lottery contest with three players; however, they found it to be exceedingly difficult to obtain an analytic Stackelberg solution for more than three players. They instead determined the optimal solutions numerically for more than

\footnotetext{
${ }^{1}$ In perfectly discriminative contests, such as the all-pay auction, this relation is deterministic (see Hillman and Riley, 1989).
} 
three and less than ten players. ${ }^{2}$ We study analytically a sequential lottery contest (in which players choose their expenditures one by one and a lottery is held after the last player has made his or her choice of expenditure) with $n$ players.

Although an analytic solution has not been available for the case of more than three players, some of its applications have been noted in the literature. For instance, Dixit (1987) noted that a sequential contest is equivalent to a Stackelberg competition between firms in which the elasticity of demand is equal to one. ${ }^{3}$ Furthermore, it has been shown that with endogenous entry and incomplete information or asymmetric players a contest with a sequential order of moves may arise (Leininger 1993; Morgan 2003). In politics, the institutional structure may also affect the contest structure. For example, lobbyists will reveal information on some issue to a politician all at once, or alternatively each piece of information will be revealed on arrival. Consequently, two different types of contest may arise. In the latter case, lobbyists may sequentially exert effort to obtain and provide information in order to influence the politician. In general, there are various types of contests, such as rent seeking, that are organized by a planner so as to determine the order of moves, with the goal of maximizing some objective, such as aggregate effort.

The model presented here examines a specific aggregative game. Aggregative games are a class of games in which each player's payoff depends only on her own

\footnotetext{
${ }^{2}$ Leininger (1991) studied a patent race using a sequential asymmetric all-pay auction under complete information, while Konrad and Leininger (2007) characterized the equilibrium of the all-pay auction under complete information with multiple players and two stages. Segev and Sela $(2011 ; 2014)$ studied a sequential asymmetric all-pay auction contest with private information for the cases of two players and multiple players. For a detailed review of contests, such as the tug-of-war and the best of $n$ contest, which involve multiple sequential battles and simultaneously exerted efforts in each battle, see Konrad (2016). For a two-stage contest between groups, see, for example, Balart at el. (2016).

${ }^{3}$ Equilibrium in a Stackelberg oligopoly has been studied in the Industrial Organization literature but under the simplifying assumption of a linear demand curve (Anderson and Engers, 1992).
} 
strategy and on the sum of all players' strategies and therefore this class of games clearly includes imperfectly discriminative contests. There is a large literature on aggregative games and their solution. Within this literature, which focuses on simultaneous games, the work of Cornes and Hartley $(2003 ; 2005)$ is closest to the analysis presented here. They utilize the notion of "replacement correspondence" associated with aggregative games to study simultaneous contests. ${ }^{4}$ Loosely speaking, they replace each player's choice variable i.e., her own effort, with the aggregate expenditure in the contest. This is a direct result of the Nash assumption, whereby a player views other players' efforts as given, and therefore, from her point of view, her investment determines the aggregate investment in the contest.

The replacement correspondence technique is applied here in a novel fashion in order to show that in a sequential lottery contest with $n$ players there exists a unique Subgame Perfect Equilibrium (henceforth: SPE) in pure strategies. We also show that, beyond some number of participants, the aggregate expenditure in this contest is less than in the corresponding simultaneous contest. In view of the fact that Glazer and Hassin (2000) have shown that in a sequential lottery contest with a small number of players aggregate expenditure is larger than in the corresponding simultaneous contest, our result regarding large contests may be surprising. ${ }^{5}$ This situation arises because, in a sequential lottery contest, aggregate expenditure is monotonically

\footnotetext{
${ }^{4}$ McManus (1962) and Selten (1970) independently exploited the basic idea underlying the replacement correspondence to establish the existence of equilibrium in a Cournot oligopoly model. For later references to the replacement correspondence, see Phlips (1995), Novshek and Sonnenschein (1978), Novshek (1984), Novshek (1985), Kukushkin (1994), Okuguchi (1993) and Corchon (1994, 1996). More recently, Cornes and Hartley apply this approach in a number of contexts, including public good provision (Cornes and Hartley 2007), contest theory and surplus sharing (Cornes and Hartley 2002, 2003 , 2005). For a comprehensive review of the literature on aggregative games, see (Cornes and Hartley 2012).

${ }^{5}$ For a study of simultaneous large all-pay auction, see Olszewski and Siegel (2016).
} 
increasing in the number of participants but the upper bound on aggregate expenditure is below the value of the prize. Thus, since there is asymptotic full rent dissipation in the simultaneous lottery contest, there exists a number of players in a contest, beyond which the aggregate expenditure in the sequential contest is lower than in the corresponding simultaneous one.

The rest of the paper is organized as follows. Section 2 presents the model of the sequential lottery contest. In section 3, an alternative problem is constructed and it is shown that it has a unique interior solution. The two main results are presented in section 4 . Section 5 concludes.

\section{The model}

There are $n$ identical risk-neutral players in a sequential lottery contest in which the order of moves is exogenous. Each player $i \in N$, observing the effort made by previous players and anticipating the future expenditures of subsequent players, invests $x_{i} \geq 0$. A lottery with one winner and one prize with a common value $v=1$ is held after all the players have made their choice. ${ }^{6}$ The probability of player $i \in N$ winning the prize is determined by the lottery contest success function:

(1) $\quad p_{i}=\left\{\begin{array}{ll}\frac{1}{n}, & \text { if } x_{1}=x_{2} \cdots=x_{n}=0 \\ \frac{x_{i}}{\sum_{j=1}^{n} x_{j}}, & \text { otherwise }\end{array}\right\}$.

Define: $\forall i \neq 0 \quad X_{i}=\sum_{j=1}^{i} x_{j}, X_{0}=0, X_{n}=X$ and $E \pi_{i}$ as player $i^{\prime}$ s expected net payoff.

\footnotetext{
${ }^{6}$ The assumption that $v=1$ is made without loss of generality.
} 
Each player $i$ solves the following problem:

(2) $\quad \max _{x_{i}} E \pi_{i}\left(x_{i} ; X_{i-1}\right)$, where $E \pi_{i}\left(x_{i} ; X_{i-1}\right)=\frac{x_{i}}{X_{i-1}+x_{i}+\sum_{j=i+1}^{n} x_{j}}-x_{i}$.

In this game, player $i$ 's pure strategy is $x_{i}:[0, \infty) \rightarrow[0, \infty)$ for $i=2, \ldots, n$, and given that investments are not made prior to the first play, the first player's strategy set is $x_{1} \in[0, \infty)$. Thus, the sum of the players' strategies is $X \in[0, \infty)$ and an SPE in pure strategies is a strategy profile $\underline{x}=\left(x_{1}, \ldots, x_{n}\right)$ such that $x_{i} \in \arg \max _{x_{i}} E \pi_{i}\left(x_{i} ; X_{i-1}\right)$, where player $i$ anticipates $x_{i+1}, \ldots, x_{n} \forall i \in N .7$

The contest can be viewed as a game in which player 1 purchases a number of sweepstakes tickets at a cost of $\$ 1$ each. Player 2 observes her choice and purchases her tickets, also at a cost of $\$ 1$ each, and so on. After all $n$ players have bought their tickets, a single winning ticket is drawn, and the owner is awarded some prize with a common value. Notice however that in the game presented here, a player can buy a fraction of a ticket. An example of this type of game is crowdsourcing. Consider, for example, a contest in which a firm offers a prize for the best logo uploaded to its website by a certain date. In such a contest, an individual can view the designs uploaded by other players prior to uploading her own. This example is similar to the model presented here, but, in order to keep the analysis tractable, we introduce the simplifying assumptions of complete information, exogenous order of players and a

\footnotetext{
7 Below we show that in each subgame of the original game there exists a unique SPE in pure strategies and thus each player can accurately anticipate the efforts made by subsequent players.
} 
specific lottery functional form. As is well-known, even this basic setting involves analytical difficulties.

\section{The alternative problem}

\subsection{The setting}

In what follows, we define, by recursion, a function $X_{i}(X)$. It is used to present the alternative problem, which is shown to be equivalent to the original problem.

The function $X_{i}(X)$ is defined as follows:

(3) $\quad X_{n}(X)=X$,

and

(4) $\quad X_{i-1}(X)=X_{i}(X)+X_{i}^{\prime}\left(X^{2}-X\right)$, where $X_{i}^{\prime}=\frac{\partial X_{i}(X)}{\partial X} \forall i \in\{2, \ldots, n\}$.

Lemma 1 specifies the structure of the function $X_{i}(X)$.

Lemma 1: $X_{i}(X)=\sum_{j=1}^{n-i+1} a_{j i} X^{j} \forall i=1, \cdots, n$, where $_{j i} \neq 0 \forall 2 \leq j \leq n$.

The proofs of the lemmas and the propositions appear in the appendix.

For a given $X_{i-1}, E \tilde{\pi}_{i}\left(X ; X_{i-1}\right)$ is defined as: ${ }^{8}$

(5) $\quad E \tilde{\pi}_{i}\left(X ; X_{i-1}\right)=\frac{X_{i}(X)-X_{i-1}}{X}-\left(X_{i}(X)-X_{i-1}\right)$.

The FOC for maximization of $E \tilde{\pi}_{i}$ with respect to $X$ is:

${ }^{8}$ Note that, by definition, $X \geq X_{i-1}$. 
(6) $\quad \frac{\partial E \tilde{\pi}_{i}}{\partial X}=\frac{X_{i}^{\prime} X+X_{i-1}-X_{i}(X)}{X^{2}}-X_{i}^{\prime}=0 \forall i$

Notice that by rearranging the terms in (6) we obtain (4).

Lemma 2 specifies the structure of the function $E \tilde{\pi}_{i}(X, 0)$.

Lemma 2: $E \tilde{\pi}_{i}(X ; 0)$ has the following structure:

(i) $E \tilde{\pi}_{i}(X ; 0)$ is a polynomial of degree $n-i+1$ with $n-i+1$ roots over the interval $[0,1]$ and $n-i$ extrema in between them, which are the roots of $E \tilde{\pi}_{i-1}(X ; 0)$.

(ii) Denote by $X_{i s}^{0}$ and $X_{i l}^{0}$ the smallest and largest roots, respectively, of $E \tilde{\pi}_{i}(X ; 0)$ over the interval $(0,1)$. Then, if $n-i$ is odd, $E \tilde{\pi}_{i}(X ; 0)$ achieves a maximum over the interval $\left(0, X_{i s}^{0}\right)$ as well as over the interval $\left(X_{i l}^{0}, 1\right)$. If $n-i$ is positive and even, then $E \tilde{\pi}_{i}(X ; 0)$ achieves a minimum over the interval $\left(0, X_{i s}^{0}\right)$ and a maximum over the interval $\left(X_{i l}^{o}, 1\right)$.

For example, $E \tilde{\pi}_{n-1}(X ; 0)=X-X^{2}$ has two roots: $X=0$ and $X=1$ and a unique maximum at $X=0.5$, where $E \tilde{\pi}_{n-2}(X ; 0)=-2 X^{3}+3 X^{2}-X$ has three roots: $X=0, X=0.5$ and $X=1$, and two extrema: a minimum over the interval $(0,0.5)$ and a maximum over the interval $(0.5,1)$ and so on. The structure of $E \tilde{\pi}_{i}(X ; 0)$ for $n-4 \leq i \leq n-1$ is illustrated in Figure 1. 


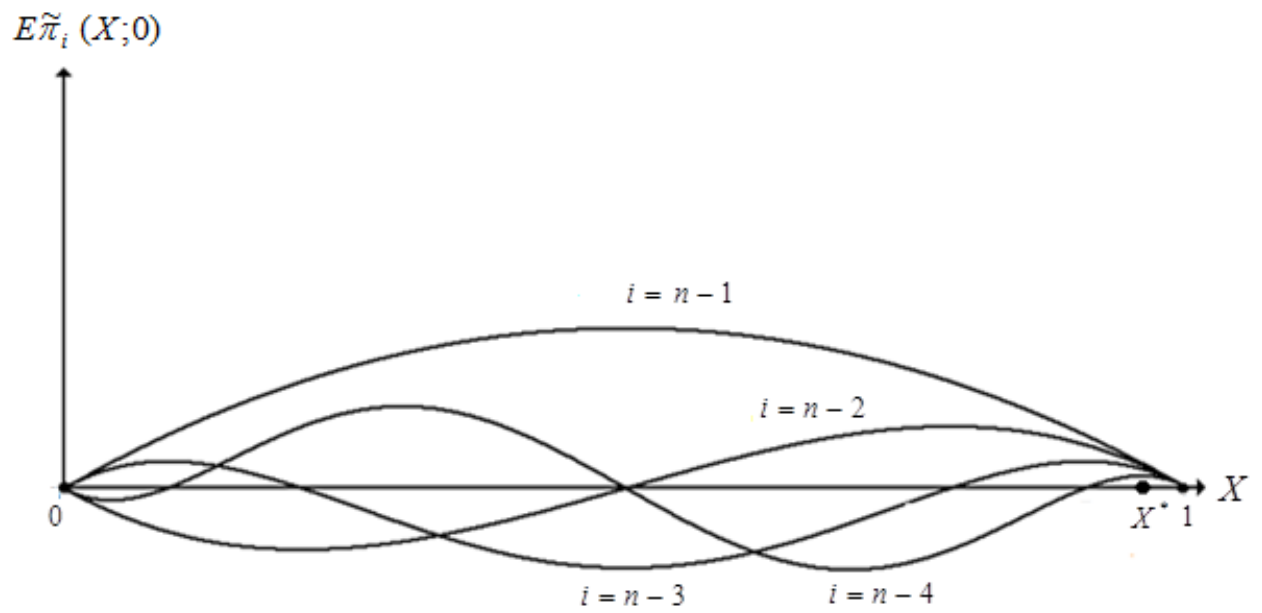

Figure 1: The functions $E \tilde{\pi}_{i}(X ; 0)$ over the interval $[0,1]$ for $i=n-4, \ldots, n-1$.

\subsection{The solution of the alternative problem}

For a given $X_{i-1}<1$, consider the following alternative problem.

(7) $\quad \max _{X} E \tilde{\pi}_{i}\left(X ; X_{i-1}\right)$

s.t. $\quad X_{i}(X)-X_{i-1} \geq 0$,

$X_{j}(X)-X_{j-1}(X) \geq 0 \forall j>i$

and

$X \leq 1$

The objective function of the alternative problem is a polynomial function of $X$. Proposition 1 utilizes both Lemma 1 and Lemma 2 to show that the alternative problem has a unique solution.

Proposition 1: Problem (7) has a unique interior solution for all $i$. Let $X^{*}$ be the solution of (7) for $i=1$. Then, given that $X_{i-1}=X_{i-1}\left(X^{*}\right), X^{*}$ is also the solution of (7) for all $i>1$.

Taking into account the behavior of $E \tilde{\pi}_{i}\left(X ; X_{i-1}\right)$ with respect to $X_{i-1}$, we utilize the special structure of $E \tilde{\pi}_{i}(X ; 0)$ described in Lemma 2 to prove Proposition 1 . The 
solution of (7) for $n=5$ and $i=1$, which is the unique maximum of $E \tilde{\pi}_{1}(X ; 0)$ over the $\operatorname{interval}\left(X_{1 l}^{o}, 1\right)$, is illustrated in Figure 1.

\section{The solution of the original problem}

This section presents the main results as follows: Proposition 2 establishes the equivalence between the alternative problem (7) and the player's original problem (2) and thus, in a sequential lottery contest with $n$ players there exists a unique SPE equilibrium in pure strategies. Lemma 3 states that under-dissipation occurs in sequential contests with $n$ players, and Proposition 3 asserts that beyond some number of participants, the aggregate expenditure in a sequential contest is even smaller than in the corresponding simultaneous contest. We present Lemma 3 first since it is needed to prove Proposition 2 which follows it:

Lemma 3: An SPE in pure strategies of the sequential lottery contest with n players satisfies:

(i) $x_{i}>0 \forall i \in N$ and (ii) $X=\sum_{j=1}^{n} x_{j} \in(0,1)$.

Proposition 2 demonstrates the link between the original and the alternative problem.

Proposition 2: Problem (7) can be rewritten as the original problem (2) for all $i$, and thus, in a sequential lottery contest, there exists a unique interior SPE in pure strategies, in which the solution to problem (7), i.e. $X^{*}$, is the aggregate investment in problem (2) and player i's investment is $x_{i}^{*}=X_{i}\left(X^{*}\right)-X_{i-1}\left(X^{*}\right)$ for all $i$.

Szidarovszky and Okuguchi (1997) have shown that, in a class of imperfectly discriminative simultaneous contests, there exists a unique Nash equilibrium in pure 
strategies. To the best of our knowledge, Proposition 2 establishes for the first time the existence of a unique SPE in pure strategies in an imperfectly discriminative sequential contest with more than three players. This was accomplished by extending the replacement correspondence technique to a dynamic setting. This extension is made possible due to the following: In each subgame of the original game, in which the aggregate investment prior to a particular play is given, there exists a unique SPE in pure strategies. Since this is anticipated, each player, given the aggregate investment prior to her play, determines the aggregate investment in the contest by choosing the level of her own investment. Therefore, in each subgame of the original game, instead of analyzing the original player's problem, a player analyzes the problem in which the choice variable is aggregate investment. Specifically, the objective function of this problem is a mapping from the set of aggregate investments to the set of a player's expected net payoffs in equilibrium. The equivalent problem is useful, since it restricts the analysis to a small set of aggregate investments, in which each player's investment is non-negative.

In order to compare the aggregate investment in the sequential lottery contest to that in the corresponding simultaneous contest, it is necessary to show that the equilibrium is unique. The amount of rent dissipation in a contest is important since it measures social loss in rent seeking contests or the designer revenue in other contests (see Myerson and Wärneryd, 2006).

In the following, we add a second lower index to denote the number of players in the contest. In particular, $X_{k, k}=X_{k}$ is the aggregate investment in a contest with $k$ players. Lemma 4 states that aggregate investment is increasing in $k$ : 
Lemma 4: $X_{n+1}^{*}>X_{n}^{*} \forall n \in N$.

Let $X_{n, S i m}^{*}$ be the aggregate expenditure in a simultaneous contest and $X_{n, S e q}^{*}$ in a sequential contest, both with $n$ participants. We can then state the following proposition:

Proposition 3: $\lim _{n \rightarrow \infty} X_{n, S e q}^{*}<1$ and thus $\exists \bar{n}$ such that $X_{n, S i m}^{*}>X_{n, S e q}^{*}$ for all $n>\bar{n}$.

Glazer and Hassin (2000) have shown that $X_{n, \text { Sim }}^{*}<X_{n, \text { Seq }}^{*}$ for $n \leq 9$. However, since only in the simultaneous lottery contest is there asymptotic full rent dissipation, if $n$ is large enough, then $X_{n, S i m}^{*}>X_{n, S e q}^{*}$. This result can be attributed to the asymmetric nature of sequential contests, since asymmetry in contests usually reduces dissipation.

\section{Conclusion}

We extend the replacement correspondence notion to a dynamic setting in order to show that, in a sequential lottery contest with $n$ players, there exists a unique SPE in pure strategies, and if $n$ is sufficiently large, then the aggregate expenditure in this contest is lower than in the corresponding simultaneous one.

\section{Acknowledgements}

We thank the audiences at the meeting of the Public Choice Society for useful discussion. All remaining errors are the authors'. Doron Klunover thanks the Max Planck Institute of Tax Law and Public Finance for its support and hospitality (part of this version of the paper was written during his visit to the Institute). 


\section{Appendix}

Proof of Lemma 1: The proof is by backwards induction. By definition, $X_{n}(X)=X$. Thus, for $i=n, X_{n}(X)=\sum_{j=1}^{n-i+1} a_{j n} X^{j} \quad$ is satisfied. Assuming that for $i=m+1$ $X_{m+1}(X)=\sum_{j=1}^{n-m} a_{j m+1} X^{j}$ and substituting $X_{m+1}(X)$ into the RHS of (4) results in:

(A.1) $X_{m}(X)=\sum_{j=1}^{n-m+1} a_{j m} X^{j}$, where $a_{j m}=(j-1)\left(a_{j-1 m+1}-a_{j m+1}\right)$ and $a_{n-m+1 m+1}=a_{0 m+1}=0$.

Thus, by backwards induction, $X_{i}(X)=\sum_{j=1}^{n-i+1} a_{j i} X^{j} \quad \forall i=1, \cdots, n$.

Proof of Lemma 2(i): The proof is by backwards induction. For $i=n-1$, from (3), (4) and (5) it follows that $E \tilde{\pi}_{n-1}(X ; 0)=X-X^{2}$ and therefore it has two roots: $X=0$ and $X=1$ over the interval $[0,1]$ and achieves a unique maximum in between them at $X=0.5 .{ }^{9}$ That is, for $i=n-1$ lemma 2(i) holds. Assume that Lemma 2(i) holds for $i=m+1$, i.e., $E \tilde{\pi}_{m+1}(X ; 0)$ is a polynomial of degree $n-m$ with $n-m$ roots over the interval $[0,1]$ and $n-m-1$ extrema in between them. In the following, it is proven that, if Lemma 2(i) is true for $i=m+1$, then it is true for $i=m$ as well. Then, by applying backwards induction the proof is completed.

Substituting $X_{i-1}=0$ in (5) and applying Lemma 1 results in:

${ }^{9}$ Notice that $\tilde{\pi}_{n}(X ; 0)=1-X$ and $\frac{\partial E \tilde{\pi}_{n}(X ; 0)}{\partial X}=-1$. 
(A.2) $E \tilde{\pi}_{i}(X ; 0)=\left(\frac{1}{X}-1\right) X_{i}(X)=(1-X) \sum_{j=1}^{n-i+1} a_{j i} X^{j-1} \forall i=1, \cdots, n$

Substituting (4) (or (3) in the case that $i=n$ ) into (6) results in:

(A.3) $\frac{\partial E \tilde{\pi}_{i}\left(X ; X_{i-1}\right)}{\partial X}=\frac{X_{i-1}-X_{i-1}(X)}{X^{2}} \quad \forall i>1$.

The LHS of (A.2) and (A.3) imply that over the interval $(0,1)$ :

(A.4) $\operatorname{sign} E \tilde{\pi}_{i-1}(X ; 0)=\operatorname{sign} X_{i-1}(X)=-\operatorname{sign} \frac{\partial E \tilde{\pi}_{i}(X ; 0)}{\partial X} \forall i>1$.

Equation (A.4) implies that $E \tilde{\pi}_{m}(X ; 0)<0$ iff $E \tilde{\pi}_{m+1}(X ; 0)$ is increasing in $X$, $E \tilde{\pi}_{m}(X ; 0)>0$ iff $E \tilde{\pi}_{m+1}(X ; 0)$ is decreasing in $X$, and $E \tilde{\pi}_{m}(X ; 0)=0$ iff $E \tilde{\pi}_{m+1}(X ; 0)$ reaches an extremum. From the RHS of (A.2), it follows that $E \tilde{\pi}_{m}(X ; 0)$ is a polynomial of degree $n-m+1$, where two of its roots are 0 and 1 . From (A.4) it follows that if $E \tilde{\pi}_{m+1}(X ; 0)$ has exactly $n-m-1$ extrema over the interval $(0,1)$, then $E \tilde{\pi}_{m}(X ; 0)$ has exactly $n-m+1$ roots over the interval $[0,1]$ where in between the roots it is either always positive or always negative. Moreover, since $\frac{\partial E \tilde{\pi}_{m}(X ; 0)}{\partial X}$ is a polynomial of degree $n-m, E \tilde{\pi}_{m}(X)$ has exactly $n-m$ extrema between these roots. Thus, the proof is completed by applying backwards induction. QED

Proof of Lemma 2(ii): The proof is by backward induction. By Lemma 2(i) and (A.4), if $E \tilde{\pi}_{m+1}(X ; 0)$ achieves a maximum over the interval $\left(0, X_{m+1 s}^{0}\right)$ as well as over the $\operatorname{interval}\left(X_{m+1 l}^{0}, 1\right)$, then $E \tilde{\pi}_{m}(X ; 0)$ achieves a minimum over the interval $\left(0, X_{m s}^{0}\right)$ and a maximum over the interval $\left(X_{m l}^{o}, 1\right)$ and vice versa. $E \tilde{\pi}_{n-1}(X ; 0)$ achieves a unique 
maximum at $X=0.5$. Thus, by applying backward induction, the proof of Lemma 2(ii) is completed.

QED

Proof for Proposition 1: Lemma 2 implies that:

(A.5) $\left\lfloor X_{(i-1) l}^{0}, 1\right\rfloor \subset\left\lfloor X_{i l}^{0}, 1\right\rfloor$,

and

$$
E \tilde{\pi}_{j}(X ; 0) \geq 0 \forall j \geq i \text { iff } X \in\left[X_{i l}^{0}, 1\right]
$$

In addition, from (5):

(A.6) $E \tilde{\pi}_{i}\left(1 ; X_{i-1}\right)=0 \forall X_{i-1}$ and $\frac{\partial E \tilde{\pi}_{i}}{\partial X_{i-1}}=1-\frac{1}{X}<0 \quad \forall X \in(0,1)$.

For each $i>1$ and a given positive $X_{i-1}$, denote by $X_{i l}^{+}$the largest root of $E \tilde{\pi}_{i}\left(X ; X_{i-1}\right)$ over the interval $(0,1)$ and define $X_{1 l}^{+} \equiv X_{1 l}^{0}$. Equation (A.6) implies that:
(A.7) $\left\lfloor X_{i l}^{+}, 1\right\rfloor \subset\left\lfloor X_{i l}^{0}, 1\right] \forall 1<i<n$,
(A. 8) $\frac{\partial^{2} E \tilde{\pi}_{i}\left(X ; X_{i-1}\right)}{\partial X_{i-1} \partial X}=\frac{1}{X^{2}}>0$

and

(A.9) $\frac{\partial^{3} E \tilde{\pi}_{i}\left(X ; X_{i-1}\right)}{\partial X^{2} \partial X_{i-1}}=-2 \frac{1}{X^{3}}<0$.

By Lemma $2, \frac{\partial E \tilde{\pi}_{i}(X ; 0)}{\partial X}=0$ has a unique solution over the interval $\left[X_{i l}^{0}, 1\right]$. Denote this solution by $X_{i l}^{0 \max }$. Then, 
(A.10) $\frac{\partial^{2} E \tilde{\pi}_{i}(X ; 0)}{\partial X^{2}}<0 \forall X \in\left[X_{i l}^{0 \max }, 1\right] .1{ }^{10}$

From (3), (4) and (A.3), at $X=1$ we have $\frac{\partial E \tilde{\pi}_{i}\left(X ; X_{i-1}\right)}{\partial X}=X_{i-1}-1<0$ and from (A.8) at $X=X_{i l}^{o \text { max }}$ we have $\frac{\partial E \tilde{\pi}_{i}\left(X ; X_{i-1}\right)}{\partial X}>0$ for all $X_{i-1} \in(0,1)$ and $1<i<n$. Thus, by the

Intermediate Value Theorem, for each $X_{i-1} \in(0,1)$ and $1<i<n, \exists X_{i l}^{+\max }\left(X_{i-1}\right)$ such that $X_{i l}^{0 \max }<X_{i l}^{+\max }\left(X_{i-1}\right)<1$ and $X_{i l}^{+\max }\left(X_{i-1}\right)$ is a solution of $\frac{\partial E \tilde{\pi}_{i}\left(X ; X_{i-1}\right)}{\partial X}=0$ over the interval $\left.\mid X_{i l}^{0}, 1\right]$. Since for each $i<n, \frac{\partial E \tilde{\pi}_{i}(X ; 0)}{\partial X}=0$ has a unique solution over the interval $\left.\mid X_{i l}^{0}, 1\right]$, (A.8), (A.9) and (A.10) imply that for each $i>1$ and $X_{i-1} \in(0,1)$, the solution of $\frac{\partial E \tilde{\pi}_{i}\left(X ; X_{i-1}\right)}{\partial X}=0$ over the interval $\left[X_{i l}^{0}, 1\right]$ is also unique, where for $i=1$ $X_{1 l}^{+\max }$ is defined as $X_{1 l}^{0 \max }{ }^{11}$ From (A.5) it follows that $X_{i l}^{+\max } \in\left[X_{j l}^{0}, 1\right]$ for all $j>i$, and thus, for a given $X_{j-1}$ such that $\left.X_{j-1}=X_{j-1}\left(X_{i l}^{+\max }\right)\right),(\mathrm{A} .3)$ and the uniqueness of $X_{j l}^{+\max }$ for each $j>i$ imply that $X_{j l}^{+\max }\left(X_{j-1}\left(X_{i l}^{+\max }\right)\right)=X_{i l}^{+\max }$ for all $j>i$. Definition (5) implies that $\operatorname{sign}\left(X_{j}\left(X_{i l}^{+\max }\right)-X_{j-1}\left(X_{i l}^{+\max }\right)\right)=\operatorname{signE} \tilde{\pi}_{j}\left(X_{i l}^{+\max } ; X_{j-1}\left(X_{i l}^{+\max }\right)\right)>0$. That is, $X_{i l}^{+\max }$ satisfies the constraints in (7). Equations (A.5) and (A.7) imply that, if

${ }^{10}$ Notice that the RHS of (A.2) implies that $\frac{\partial E \tilde{\pi}_{i}(X ; 0)}{\partial X}$ is a polynomial of degree $n-i$ with $n-i$ roots at the extrema of $E \tilde{\pi}_{i}(X ; 0)$ and $n-i-1$ extrema in between those roots, and $\frac{\partial^{2} E \tilde{\pi}_{i}(X ; 0)}{\partial X^{2}}$ is a polynomial of degree $n-i-1$ with $n-i-1$ roots at the extrema of $\frac{\partial E \tilde{\pi}_{i}(X ; 0)}{\partial X}$ and $n-i-2$ extrema in between those roots.

${ }^{11}$ Notice that for $X_{n-1}>0, \tilde{\pi}_{n}\left(X ; X_{n-1}\right)$ is concave and thus, given that $X_{n-1}>0$, it also has a unique maximum. 
$\operatorname{signE} \tilde{\pi}_{i}\left(X ; X_{i-1}\right) \geq 0, \operatorname{signE} \tilde{\pi}_{j}\left(X ; X_{j-1}(X)\right) \geq 0 \forall j>i$ and $X \leq 1$, then $X \in\left[X_{i l}^{0}, 1\right]$. Thus, $X_{i l}^{+\max }$ is the unique solution of (7) for each $i$. In particular, denote $X_{1 l}^{+\max }=X^{*}$; then $X_{i l}^{+\max }\left(X_{i-1}\left(X^{*}\right)\right)=X^{*}$ for each $i>1$.

QED

Proof of Lemma 3: Given that $E \pi_{n}(\varepsilon ; 0)=1-\varepsilon>E \pi_{n}(0 ; 0)=\frac{1}{n}$, player $n^{\prime} s$ best

response to $X_{n-1}=0$ is $x_{n}=\varepsilon>0$, i.e., $X>0$. Furthermore, since $E \pi_{i}=x_{i}\left(\frac{1}{X}-1\right)$, $E \pi_{i}>0$ iff $X<1$ and $x_{i}>0$. Thus, player $n^{\prime} \mathrm{s}$ best response to $X_{n-1}<1$ is $x_{n}>0$ such that $X_{n-1}+x_{n}<1$. Given that player $n-1$ knows this, her optimal strategy is $x_{n-1}>0$ such that $X_{n-2}+x_{n-1}<1$. By applying backward induction, player $i$ 's optimal strategy is to invest such that the aggregate investment will be smaller than 1 .

QED

Proof of Proposition 2: The proof is by backward induction. By definition, for $i=n$, $E \tilde{\pi}_{n}=\frac{X_{n}(X)-X_{n-1}}{X}-\left(X_{n}(X)-X_{n-1}\right)=\frac{X-X_{n-1}}{X}-\left(X-X_{n-1}\right)=\frac{x_{n}}{X}-x_{n}=E \pi_{n} \forall X_{n-1}>0$.

That is, for a given $X_{n-1}>0$, (2) can be rewritten as (7). Assume that for $i=k,(2)$ can be rewritten as (7). Proposition 1 and (A.3) imply that (7) has a unique interior solution that satisfies $X_{k-1}=X_{k-1}(X)$. Thus, for player $i=k-1$ who takes this into account, $E \tilde{\pi}_{k-1}=\frac{X_{k-1}(X)-X_{k-2}}{X}-\left(X_{k-1}(X)-X_{k-2}\right)=\frac{X_{k-1}-X_{k-2}}{X}-\left(X_{k-1}-X_{k-2}\right)=\frac{x_{k-1}}{X}-x_{k-1}=E \pi_{k-1}$, where $X$ satisfies $X_{k-1}(X)-X_{k-2} \geq 0$ and the constraints in (7) for $i=k$. That is, for 
$i=k-1$, (2) can be rewritten as (7). Thus, by backward induction, (2) can be rewritten as (7) for each $i$, and the solution of (7) for $i=1$, i.e., $X^{*}$, is the unique aggregate investment equilibrium in the contest, where player $i$ 's investment in equilibrium is $x_{i}^{*}=X_{i}\left(X^{*}\right)-X_{i-1}\left(X^{*}\right)$ for each $i$. Notice that, by lemma 3 , the assumptions that $X_{n-1}>0, \quad X_{i-1}<1$ for each $i=1, \ldots, n-1, \quad$ and $X \leq 1$ are not binding. QED

Proof of Lemma 4: By definition, $E \tilde{\pi}_{1, n-1}(X) \equiv E \tilde{\pi}_{2, n}(X ; 0)$. From the proof of Proposition 1 and Proposition 2, the aggregate equilibrium investment in contests with $n-1$ players is the maximum of $E \tilde{\pi}_{2, n}(X ; 0)$ over the open interval $\left(X_{2 l, n}^{0}, 1\right)$ and with $n$ players is the maximum of $E \tilde{\pi}_{1, n}(X ; 0)$ over the open interval $\left(X_{1 l, n}^{0}, 1\right)$. Thus, (A.5) implies that $X_{n-1}^{*}<X_{n}^{*} \forall n \in N$.

QED

Proof of Proposition 3: From (3) and (4), $X_{i, n}(1)=1 \forall i, n$. Thus, from the LHS of (6), $\frac{\partial E \tilde{\pi}_{1, n}(1)}{\partial X_{n}}=-1 \forall n$. Thus, $\lim _{n \rightarrow \infty} \frac{\partial E \tilde{\pi}_{1, n}(1)}{\partial X_{n}} \neq 0$ and $\lim _{n \rightarrow \infty} X_{n, S e q}^{*} \neq 1$. By the SOC for maximization and lemma $4, \lim _{n \rightarrow \infty} X_{n, S e q}^{*}=1-\varepsilon$, where $0<\varepsilon<1$. In a simultaneous contest, $E \pi_{i, n}\left(X_{n} ; X_{-i, n}\right)=\frac{X_{n}-X_{-i, n}}{X_{n}}-\left(X_{n}-X_{-i, n}\right) \forall i$, where $X_{-i, n}=\sum_{j \neq i}^{j=n} x_{j, n}$. In the symmetric Nash equilibrium, $\frac{\partial E \pi_{i, n}\left(X_{n}\right)}{\partial X_{n}}=\frac{n-1}{n X_{n}}-1=0 \quad \forall i$. Thus, $X_{n, \operatorname{Sim}}^{*}=\frac{n-1}{n}$, 
$\lim _{n \rightarrow \infty} X_{n, S i m}^{*}=1$ and $\lim _{n \rightarrow \infty} \frac{\partial E \pi_{1, n}(1)}{\partial X_{n}}=\lim _{n \rightarrow \infty} \frac{n-1}{n}-1=0$. Therefore, $\exists \bar{n}$ such that $X_{n, \text { Seq }}^{*}<1-\varepsilon<X_{n, \text { Sim }}^{*}<1 \forall n>\bar{n}$. QED 


\section{References}

Anderson, S.P., Engers, M., 1992. Stackelberg versus cournot oligopoly equilibrium. International Journal of Industrial Organization, 10, 127-135.

Baik, K.H., Shogren, J.F., 1992. Strategic behavior in contests: comment. The American Economic Review, 82(1), 359-362.

Balart, P., Flamand, S., Gürtler, O., Troumpounis, O., 2016. Sequential choice of sharing rules in collective contests. Unpublished manuscript.

Corchon, L., 1994. Comparative statics for aggregative games: the strong concavity case. Mathematical Social Sciences, 28, 151-165.

Corchon, L., 1996. Theories of imperfectly competitive markets. Springer-Verlag

Cornes, R., Hartley, R., 2002. Joint production games with mixed sharing rules. Keele Economics Research Papers 2002/16. UK

Cornes, R., Hartley, R., 2003. Risk aversion, heterogeneity and contests. Public Choice, 117, 1-25.

Cornes, R., Hartley, R., 2005. Asymmetric contests with general technologies. Economic Theory, 26, 923-946.

Cornes, R., Hartley, R., 2007. Aggregative public good games. Journal of Public Economic Theory, 9, 201-219.

Cornes, R., Hartley, R., 2012. Fully aggregative games. Economics Letters, 116, 631-633.

Dixit, A., 1987. Strategic behavior in contests. American Economic Review, 77, 891-898.

Esteban, J., Ray, D., 2011. Linking conflict to inequality and polarization. American Economic Review, 101, 1345-1374.

Fullerton, R.L., McAfee, R.P., 1999. Auctioning entry into tournaments. Journal of Political Economy, 107, 573-605.

Glazer, A., Hassin, R., 2000. Sequential rent seeking. Public Choice, 102, 219-228.

Hillman, A.L., 2013. Rent seeking. In Reksulak, M., Razzolini, L., Shughart, W.F. II (Eds.), The Elgar Companion to Public Choice (2nd Edition). Edward Elgar, Cheltenham U.K., pp. 307-330. 
Hillman, A.L., Riley, J.G., 1989. Politically contestable rents and transfers. Economics $\mathcal{E}$ Politics, 1(1), 17-39.

Konrad, K.A., 2009. Strategy and Dynamics in Contests. Oxford University Press, Oxford UK.

Konrad, K.A., 2016. Budget and Effort Choice in Sequential Colonel Blotto Campaigns. Unpublished manuscript.

Konrad, K.A., Leininger, W., 2007. The generalized Stackelberg equilibrium of the all-pay auction with complete information. Review of Economic Design, 11, 165-174.

Kukushkin, N.S., 1994. A fixed-point theorem for decreasing mappings. Economics Letters, 46, 23-26.

Leininger, W., 1991. Patent competition, rent dissipation and the persistence of monopoly. Journal of Economic Theory, 53, 146-172.

Leininger, W., 1993. More efficient rent-seeking - A Münchhausen solution. Public Choice, 75, 433-462.

Leininger, W., Yang, C.L., 1994. Dynamic rent-seeking games. Games and Economic Behavior, 7, 406-427.

Linster., G.B., 1993. Stackelberg rent-seeking. Public Choice, 77, 307-321.

McManus, M., 1962. Equilibrium, numbers and size in Cournot oligopoly. Bulletin of Economic Research, 16, 68-75.

Morgan, J., 2003. Sequential contests. Public Choice, 116, 1-18.

Morgan, J., Várdy, F., 2007. The value of commitment in contests and tournaments when observation is costly. Games and Economic Behavior, 60(2), 326-338.

Myerson, R.B., Wärneryd, K., 2006. Population uncertainty in contests. Economic Theory, 27(2), 469-474.

Novshek, W., 1984. Finding all n-firm Cournot equilibria. International Economic Review, 25, 61-70.

Novshek, W., 1985. On the existence of Cournot equilibrium. Review of Economic Studies, 52, 85-98.

Novshek, W., Sonnenschein, H., 1978. Cournot and Walras equilibrium. Journal of 
Economic Theory, 19, 223-266.

Okuguchi, K., 1993. Unified approach to Cournot models: oligopoly, taxation and aggregate provision of a pure public good. European Journal of Political Economy, 9, 233-245.

Olszewski, W., Siegel, R., 2016. Large contests. Econometrica, 84, 835-854.

Phlips, L., 1995. Competition Policy: A game-theoretic perspective. Cambridge University Press.

Segev, E., Sela, A., 2011. Sequential all-pay auctions with head starts. Social Choice and Welfare, 43, 1-31.

Segev, E., Sela, A., 2014. Multi-stage sequential all-pay auctions. European Economic Review, 70, 371-382.

Selten, R., 1970. Preispolitik der Mehrproduktenunternehmung in der Statischen Theorie. Springer-Verlag

Serena, M., 2017. Sequential contests revised. SSRN Working Paper https://papers.ssrn.com/sol3/papers.cfm?abstract_id=2938412

Skaperdas, S., 1996. Contest success functions. Economic Theory, 7, 283-290.

Szidarovszky, F., Okuguchi, K., 1997. On the existence and uniqueness of pure Nash equilibrium in rent-seeking games. Games and Economic Behavior, 18,135-140.

Tullock, G., 1980. Efficient rent seeking. In J.M. Buchanan, R.D. Tollison and G. Tullock (Eds.), Towards a theory of the rent-seeking society, 97-112. College Station: Texas A\&M University Press.

Wärneryd, K., 2003. Information in conflicts. Journal of Economic Theory, 110, 121-136.

Yildirim, H., 2005. Contests with multiple rounds. Games and Economic Behavior, 51(1), 213-227. 